\title{
Phenolic extracts of Rubus ulmifolius Schott flowers: characterization, microencapsulation and incorporation into yogurts as nutraceutical sources
}

Received 31st December 2013

Accepted 1st March 2014

DOI: $10.1039 / c 3 f \circ 60721 f$

www.rsc.org/foodfunction
Ana Martins, ${ }^{\text {ac }}$ Lillian Barros, ${ }^{\text {ab }}$ Ana Maria Carvalho, ${ }^{a}$ Celestino Santos-Buelga, ${ }^{b}$ Isabel P. Fernandes, ${ }^{C}$ Filomena Barreiro ${ }^{C}$ and Isabel C. F. R. Ferreira*a

Rubus ulmifolius Schott (Rosaceae), known as wild blackberry, is a perennial shrub found in wild and cultivated habitats in Europe, Asia and North Africa. Traditionally, it is used for homemade remedies because of its medicinal properties, including antioxidant activity. In the present work, phenolic extracts of R. ulmifolius flower buds obtained by decoction and hydroalcoholic extraction were chemically and biologically characterized. Several phenolic compounds were identified in both decoction and hydroalcoholic extracts of flowers, ellagitannin derivatives being the most abundant ones, namely the sanguiin H-10 isomer and lambertianin. Additionally, comparing with the decoction form, the hydroalcoholic extract presented both higher phenolic content and antioxidant activity. The hydroalcoholic extract was thereafter microencapsulated in an alginate-based matrix and incorporated into a yogurt to achieve antioxidant benefits. In what concerns the performed incorporation tests, the obtained results pointed out that, among the tested samples, the yoghurt containing the microencapsulated extract presented a slightly higher antioxidant activity, and that both forms (free and microencapsulated extracts) gave rise to products with higher activity than the control. In conclusion, this study demonstrated the antioxidant potential of the $R$. ulmifolius hydroalcoholic extract and the effectiveness of the microencapsulation technique used for its preservation, thus opening new prospects for the exploitation of these natural phenolic extracts in food applications.

\section{Introduction}

Reactive oxygen and nitrogen species are formed during normal cellular metabolism, but when presented in high concentrations they become toxic, this effect being related to several chronic diseases such as cancer, cardiovascular and neurodegenerative diseases. ${ }^{1,2}$ Exposure to those species from a variety of sources has led the organism to develop defense mechanisms (endogenous defenses) in order to protect the cells against excessive levels of free radicals. Antioxidant defenses can be enzymatic and nonenzymatic. Examples of enzymatic defenses are superoxide dismutase (SOD), catalase (CAT), glutathione peroxidase (GSH-Px), and glutathione reductase (GSH-R). ${ }^{2,3}$ The endogenous nonenzymatic antioxidant defenses include glutathione (GSH), $\alpha$ tocopherol (vitamin E), ascorbic acid (vitamin C) and lipoic acid. ${ }^{\mathbf{1 , 3}}$

${ }^{a}$ Mountain Research Center (CIMO), ESA, Polytechnic Institute of Bragança, Campus Santa Apolónia Ap. 1172, 5301-855 Bragança, Portugal. E-mail: iferreira@ipb.pt; Fax: +351-273-325405; Tel: +351-273-303219

${ }^{b}$ GIP-USAL, Faculty of Pharmacy, University of Salamanca, Campus Miguel de Unamuno, 37007 Salamanca, Spain

${ }^{c}$ Laboratory of Separation and Reaction Engineering (LSRE), Associate Laboratory LSRE/LCM, Polytechnic Institute of Bragança, Campus Santa Apolónia Ap. 1134, 5301-857 Bragança, Portugal
Exogenous antioxidant defenses supplied by diet have gained special interest, namely the use of phenolic compounds from plants. In fact, plants are natural sources of effective bioactive phenolic compounds. Beneficial activities of these compounds include risk reduction of cardiovascular and neurodegenerative diseases, diabetes or osteoporosis. The slower progression of certain cancers is another benefit, enabling plant polyphenols to be potential chemopreventive and anti-cancer agents in humans. ${ }^{4}$

Rubus ulmifolius Schott (Rosaceae), known as wild blackberry, is a perennial shrub found in wild and cultivated habitats in Europe, Asia and North Africa. ${ }^{5}$ Traditionally, R. ulmifolius is regarded as an interesting medicinal plant and considered to be anticatarrhal, antiseptic, diuretic, anti-inflammatory, antioxidant, astringent, and antispasmodic. ${ }^{6,7}$ Decoctions from dry flower buds are used for diarrhea, menstrual pain, menopause disorders, liver diseases, aphtha, gingivitis, hypertension and diabetes. $^{8}$

The antioxidant properties of the methanolic extract from $R$. ulmifolius flowers were previously reported, ${ }^{9}$ but not for the hydroalcoholic extract or for its most used form, decoction. The mentioned extracts could be included in formulations of nutraceuticals or functional foods due to their attractive bioactive properties. 
Microencapsulation is a technique that allows bioactive compounds/extracts to be incorporated into a matrix or a coating shell in the form of microparticles with diameters ranging from 1 to 1000 micrometers. ${ }^{10}$ These microparticles can release their contents along with time by means of different release mechanisms, which are dependent on the encapsulation materials used, production process, final morphology and applications. This technology has been used in several fields including pharmaceutical, food and cosmetic industries. Encapsulation of natural extracts can provide protection against the action of atmospheric agents (light, moisture and heat), ensuring an increase of their stability and thus control of their bioavailability. ${ }^{10}$ There are several documented examples of the application of this technique with natural extracts for production of functional foods. For example, Krishnaiah et al. ${ }^{\mathbf{1 1}}$ studied the Morinda citrifolia L. fruit extract encapsulation in $\kappa$-carrageenan and maltodextrin matrices. This extract is recognized for its antibiotic and antioxidant properties due to the presence of high phenolic compound content. In addition, microcapsule production from cactus pear fruit (Opuntia ficus-indica) extracts represents an interesting food additive due to the presence of antioxidants and as a red colorant. ${ }^{12}$

The effective incorporation of microencapsulated natural extracts into foods was performed by Çam et al. ${ }^{13}$ and Ezhilarasi et al. ${ }^{14}$ by testing the incorporation of microencapsulated Punica granatum L. peel and Garcinia cowa Roxb. fruit extracts in icecream and bread, respectively.

The present study aims to characterize the phenolic compounds present in the hydroalcoholic extract and decoction of $R$. ulmifolius flower buds and to evaluate their antioxidant potential. Furthermore, the hydroalcoholic extract in its lyophilized form was microencapsulated in an alginate matrix by an atomization/coagulation technique. Additionally, an equivalent amount of $R$. ulmifolius hydroalcoholic extract, free and microencapsulated, was added to yogurt samples and its antioxidant activity was evaluated and compared with a control. The results obtained showed the antioxidant potential of the R. ulmifolius hydroalcoholic extract and the effectiveness of the microencapsulation technique to preserve the antioxidant activity, thus opening new prospects for the exploitation of these natural phenolic extracts for nutraceutical applications.

\section{Experimental}

\section{Plant materials}

Samples of flower buds from different specimens of Rubus ulmifolius Schott selected randomly were collected in late spring of 2009, in the Natural Park of Montesinho territory, Trás-osMontes, North-eastern Portugal, considering the Portuguese folk pharmacopoeia, the local medicinal criteria of use and the plant growth patterns. Morphological key characters from the Flora Iberica $^{\mathbf{1 5}}$ were used for plant identification. Voucher specimens are deposited in the Herbário da Escola Superior Agrária de Bragança (BRESA). The samples were lyophilized (FreeZone 4.5, Labconco, Kansas, USA), reduced to powder ( $\sim 20$ mesh) and kept under the best conditions for subsequent use.

\section{Standards and reagents}

HPLC-grade acetonitrile was obtained from Merck KgaA (Darmstadt, Germany). Formic acid and acetic acid were purchased from Prolabo (VWR International, France). Trolox (6-hydroxy-2,5,7,8-tetramethylchroman-2-carboxylic acid) was purchased from Matreya (PA, USA). 2,2-Diphenyl-1-picrylhydrazyl (DPPH) was obtained from Alfa Aesar (Ward Hill, MA, USA). Phenolic compound standards were from Extrasynthèse (Genay, France). Alginic acid sodium was obtained from Fluka Chemie and calcium chloride 2-hydrate was purchased from Panreac Química S.A.U.

All other chemicals were of analytical grade and purchased from chemical suppliers. Water was treated in a Milli-Q water purification system (TGI Pure Water Systems, USA).

\section{Extraction procedures for phenolic compounds}

Hydroalcoholic extraction was performed using the lyophilized plant material $(1 \mathrm{~g})$ stirring with $30 \mathrm{~mL}$ of methanol-water (80:20, v/v) at $25{ }^{\circ} \mathrm{C}$ at $150 \mathrm{rpm}$ for $1 \mathrm{~h}$ and filtered through Whatman no. 4 paper. The residue was then extracted with one additional $30 \mathrm{~mL}$ portion of the hydroalcoholic mixture. The combined hydroalcoholic extracts were evaporated at $35{ }^{\circ} \mathrm{C}$ under reduced pressure (rotary evaporator Büchi R-210, Flawil, Switzerland) and then further lyophilized (FreeZone 4.5, Labconco, Kansas, USA).

A decoction was also prepared from the lyophilized plant material $(1 \mathrm{~g})$, by adding $200 \mathrm{~mL}$ of distilled water, heating (heating plate, VELP scientific) and boiling for $5 \mathrm{~min}$. The mixture was left to stand for $5 \mathrm{~min}$ and then filtered under reduced pressure. The obtained decoction was frozen and lyophilized.

The hydroalcoholic extract and lyophilized decoction were re-dissolved in methanol-water $(80: 20, \mathrm{v} / \mathrm{v})$ and water, respectively (final concentration: $2.5 \mathrm{mg} \mathrm{mL}^{-1}$ ), for phenolic compound determination and antioxidant activity evaluation. The final solutions were further diluted to different concentrations to be subjected to distinct in vitro assays.

\section{Characterization of the extracts in phenolic compounds}

The extracts were analysed using a Hewlett-Packard 1100 chromatograph (Agilent Technologies) with a quaternary pump and a diode array detector (DAD) coupled to an HP Chem Station (rev. A.05.04) data-processing station. A Waters Spherisorb S3 ODS-2 $\mathrm{C}_{18}, 3 \mu \mathrm{m}(4.6 \mathrm{~mm} \times 150 \mathrm{~mm})$ column thermostatted at $35{ }^{\circ} \mathrm{C}$ was used. The solvents used were: (A) $0.1 \%$ formic acid in water and (B) acetonitrile. The elution gradient established was $15 \%$ isocratic for $5 \mathrm{~min}, 15 \% \mathrm{~B}$ to $20 \% \mathrm{~B}$ over $5 \mathrm{~min}, 20-25 \%$ B over $10 \mathrm{~min}, 25-35 \%$ B over $10 \mathrm{~min}, 35-50 \%$ for $10 \mathrm{~min}$, and re-equilibration of the column, at a flow rate of $0.5 \mathrm{~mL} \mathrm{~min}^{-1}$. Double online detection was carried out using the DAD with $280 \mathrm{~nm}$ and $370 \mathrm{~nm}$ as preferred wavelengths and using a mass spectrometer (MS) connected to an HPLC system via the DAD cell outlet.

MS detection was performed using an API 3200 Qtrap (Applied Biosystems, Darmstadt, Germany) equipped with an 
ESI source and a triple quadrupole-ion trap mass analyzer that was controlled by using the Analyst 5.1 software. Zero grade air served as the nebulizer gas (30 psi) and turbo gas for solvent drying $\left(400{ }^{\circ} \mathrm{C}, 40 \mathrm{psi}\right)$. Nitrogen served as the curtain (20 psi) and collision gas (medium). The quadrupoles were set at unit resolution. The ion spray voltage was set at $-4500 \mathrm{~V}$ in the negative mode. The MS detector was programmed for recording in two consecutive modes: enhanced MS (EMS) and enhanced product ion (EPI) analyses. EMS was employed to record full scan spectra, so as to obtain an overview of all the ions in the sample. Settings used were: declustering potential (DP) $-450 \mathrm{~V}$, entrance potential (EP) $-6 \mathrm{~V}$, and collision energy (CE) $-10 \mathrm{~V}$. EPI mode was performed in order to obtain the fragmentation pattern of the parent ion(s) in the previous scan using the following parameters: $\mathrm{DP}-50 \mathrm{~V}, \mathrm{EP}-6 \mathrm{~V}, \mathrm{CE}-25 \mathrm{~V}$, and collision energy spread (CES) $0 \mathrm{~V}$. Spectra were recorded in negative ion mode between $\mathrm{m} / \mathrm{z} 100$ and 1700 .

The phenolic compounds were characterized according to their UV and mass spectra and retention times compared with standards when available. For the quantitative analysis of phenolic compounds, a 5-level calibration curve was obtained by injection of known concentrations $\left(2.5-100 \mu \mathrm{g} \mathrm{mL} \mathrm{m}^{-1}\right)$ of different standard compounds: catechin $(y=158.42 x-11.38$; $\left.R^{2}=0.9999\right) ;$ chlorogenic acid $\left(y=600.27 x-763.62 ; R^{2}=\right.$ $0.9998) ; p$-coumaric acid $\left(y=884.6 x+184.49 ; R^{2}=0.9999\right)$; ellagic acid $\left(y=32.72 x+77.8 ; R^{2}=0.9999\right)$; ferulic acid $(y=$ $\left.505.97 x-64.578 ; R^{2}=0.9999\right)$; kaempferol 3-O-glucoside $(y=$ $\left.190.75 x-36.158 ; R^{2}=1.000\right)$; kaempferol 3-O-rutinoside $\left(y=175.02 x-43.877 ; R^{2}=0.9999\right)$; quercetin 3-O-glucoside $\left(y=316.48 x-2.9142 ; R^{2}=1.000\right)$; quercetin 3-O-rutinoside $(y=$ $\left.222.79 x-243.11 ; R^{2}=0.9998\right)$. The results were expressed in mg per $100 \mathrm{~g}$ of dry weight (dw).

\section{Evaluation of in vitro antioxidant properties}

DPPH radical-scavenging activity. This methodology was performed using an ELX800 Microplate Reader (Bio-Tek, Bedfordshire, UK). The reaction mixture in each one of the 96-wells consisted of one of the different concentration solutions $(30 \mu \mathrm{L})$ and methanolic solution $(270 \mu \mathrm{L})$ containing $\mathrm{DPPH}$ radicals $\left(6 \times 10^{-5} \mathrm{~mol} \mathrm{~L}^{-1}\right)$. The mixture was left to stand for $30 \mathrm{~min}$ in the dark. The reduction of the DPPH radical was determined by measuring the absorption at $515 \mathrm{~nm}$. The radical scavenging activity (RSA) was calculated as the percentage of DPPH discolouration using the equation: $\operatorname{RSA}(\%)=\left[\left(A_{\mathrm{DPPH}}-A_{\mathrm{S}}\right) / A_{\mathrm{DPPH}}\right]$ $\times 100$, where $A_{\mathrm{S}}$ is the absorbance of the solution when the sample extract has been added at a particular level and $A_{\mathrm{DPPH}}$ is the absorbance of the DPPH solution. ${ }^{9}$ The extract concentration providing $50 \%$ antioxidant activity $\left(\mathrm{EC}_{50}\right)$ was calculated from the graph of DPPH scavenging activity against extract concentrations. Trolox was used as the standard.

Reducing power. This methodology was performed using the Microplate Reader described above. The different concentration solutions $(0.5 \mathrm{~mL})$ were mixed with sodium phosphate buffer $\left(200 \mathrm{mmol} \mathrm{L}^{-1}, \mathrm{pH} 6.6,0.5 \mathrm{~mL}\right)$ and potassium ferricyanide $(1 \%$ $\mathrm{w} / \mathrm{v}, 0.5 \mathrm{~mL}$ ). The mixture was incubated at $50{ }^{\circ} \mathrm{C}$ for $20 \mathrm{~min}$, and trichloroacetic acid $(10 \% \mathrm{w} / \mathrm{v}, 0.5 \mathrm{~mL})$ was added. The mixture $(0.8 \mathrm{~mL})$ was poured into the 48 -wells, as also was deionised water $(0.8 \mathrm{~mL})$ and ferric chloride $(0.1 \% \mathrm{w} / \mathrm{v}$, $0.16 \mathrm{~mL}$ ), and the absorbance was measured at $690 \mathrm{~nm} .{ }^{9}$ The extract concentration providing an absorbance of $0.5\left(\mathrm{EC}_{50}\right)$ was calculated from the graph of absorbance at $690 \mathrm{~nm}$ against extract concentrations. Trolox was used as the standard.

Inhibition of $\boldsymbol{\beta}$-carotene bleaching. A solution of $\beta$-carotene was prepared by dissolving $\beta$-carotene $(2 \mathrm{mg}$ ) in chloroform $(10 \mathrm{~mL})$. Two millilitres of this solution were pipetted into a round-bottom flask. After the chloroform was removed at $40{ }^{\circ} \mathrm{C}$ under vacuum, linoleic acid $(40 \mathrm{mg})$, Tween 80 emulsifier (400 $\mathrm{mg}$ ), and distilled water $(100 \mathrm{~mL})$ were added to the flask with vigorous shaking. Aliquots $(4.8 \mathrm{~mL})$ of this emulsion were transferred into different test tubes containing different concentrations of the samples $(0.2 \mathrm{~mL})$. The tubes were shaken and incubated at $50{ }^{\circ} \mathrm{C}$ in a water bath. As soon as the emulsion was added to each tube, the zero time absorbance was measured at $470 \mathrm{~nm}$ using a spectrophotometer (AnalytikJena, Jena, Germany). $\beta$-Carotene bleaching inhibition was calculated using the following equation: (abs. after $2 \mathrm{~h}$ of assay/initial abs.) $\times 100 .{ }^{9}$ The extract concentration providing $50 \%$ antioxidant activity $\left(\mathrm{EC}_{50}\right)$ was calculated from the graph of $\beta$-carotene bleaching inhibition against extract concentrations. Trolox was used as the standard.

Inhibition of lipid peroxidation using thiobarbituric acid reactive substances (TBARSs). Porcine (Sus scrofa) brains were obtained from official slaughtering animals, dissected, and homogenized with a Polytron in ice-cold Tris-HCl buffer (20 mM, pH 7.4) to produce a $1: 2(\mathrm{w} / \mathrm{v})$ brain tissue homogenate which was centrifuged at $3000 \mathrm{~g}$ for $10 \mathrm{~min}$. An aliquot $(0.1 \mathrm{~mL})$ of the supernatant was incubated with the different solution concentrations $(0.2 \mathrm{~mL})$ in the presence of $\mathrm{FeSO}_{4}$ $(10 \mu \mathrm{M} ; 0.1 \mathrm{~mL})$ and ascorbic acid $(0.1 \mathrm{mM} ; 0.1 \mathrm{~mL})$ at $37{ }^{\circ} \mathrm{C}$ for $1 \mathrm{~h}$. The reaction was stopped by the addition of trichloroacetic acid $(28 \% \mathrm{w} / \mathrm{v}, 0.5 \mathrm{~mL})$, followed by thiobarbituric acid (TBA, $2 \%, \mathrm{w} / \mathrm{v}, 0.38 \mathrm{~mL}$ ), and the mixture was then heated at $80{ }^{\circ} \mathrm{C}$ for $20 \mathrm{~min}$. After centrifugation at $3000 \mathrm{~g}$ for $10 \mathrm{~min}$ to remove the precipitated protein, the colour intensity of the malondialdehyde (MDA)-TBA complex in the supernatant was measured by its absorbance at $532 \mathrm{~nm}$. The inhibition ratio (\%) was calculated using the following formula: inhibition ratio $(\%)=[(A-$ $B) / A] \times 100 \%$, where $A$ and $B$ are the absorbance of the control and the compound solution, respectively. ${ }^{9}$ The extract concentration providing $50 \%$ antioxidant activity $\left(\mathrm{EC}_{50}\right)$ was calculated from the graph of TBARS formation inhibition against extract concentrations. Trolox was used as the standard.

\section{Microencapsulation of the $R$. ulmifolius hydroalcoholic extract}

Microspheres containing lyophilized $R$. ulmifolius hydroalcoholic extract were prepared by an atomization/coagulation technique. Briefly, sodium alginate was used as the matrix material and $\mathrm{CaCl}_{2}$ aqueous solution as the coagulation agent. A hydroalcoholic extract solution was prepared by dissolving $50 \mathrm{mg}$ of the lyophilized extract in $10 \mathrm{~mL}$ of distilled water under stirring. Then, this solution was filtered for residue 
removal and $400 \mathrm{mg}$ of sodium alginate was added. The solution was kept under stirring until complete alginate dissolution was achieved. Thereafter, the alginate solution containing the extract was atomized using a NISCO Var J30 system (feed rate of $0.3 \mathrm{~mL} \mathrm{~min}^{-1}$ and a nitrogen pressure of $0.1 \mathrm{bar}$ ) to produce the microspheres. The atomized microspheres were immediately coagulated by contacting with a $\mathrm{CaCl}_{2}$ aqueous solution $(250 \mathrm{~mL}$ at a concentration of $4 \%(\mathrm{w} / \mathrm{v}))$ for 4 hours. The resulting microspheres were collected by filtration under reduced pressure and washed twice with distilled water. The obtained microspheres were then lyophilized and stored under dark conditions at $4{ }^{\circ} \mathrm{C}$.

\section{Microsphere characterization}

Microspheres were analyzed by optical microscopy (OM) using a Nikon Eclipse 50i microscope equipped with a Nikon Digital Sight camera and NIS Elements software for data acquisition. OM analysis was applied to access the size and morphology of the microspheres after the production and coagulation stages, respectively. It was also possible to infer the presence/absence of extract inside the microspheres.

The effective extract incorporation into the alginate matrix was investigated by FTIR analysis. For that purpose, spectra of pure alginate, free hydroalcoholic extract of $R$. ulmifolius and the corresponding microspheres were collected on a FTIR Bomen (model MB 104) by preparing $\mathrm{KBr}$ pellets at a sample concentration of $1 \%(\mathrm{w} / \mathrm{w})$. Spectra were recorded at a resolution of $4 \mathrm{~cm}^{-1}$ between 650 and $4000 \mathrm{~cm}^{-1}$ by co-adding 48 scans.

The dry residue (DR) and encapsulation efficiency (EE) were also evaluated. DR was calculated as the ratio between the dry (lyophilized) and the wet microsphere weight (\%,w/w). EE evaluation was done through the quantification of the nonencapsulated extract. For that purpose the remaining extract in the coagulation and that in the first washing solution were quantified by HPLC and added. The second washing solution was found to be absent of the extract.

The encapsulation efficiency was calculated according to the following expression:

$$
\mathrm{EE}=\left[\left(M_{\mathrm{e}-\mathrm{t}}-M_{\mathrm{e}-\mathrm{ne}}\right) /\left(M_{\mathrm{e}-\mathrm{t}}\right)\right] \times 100
$$

in which $M_{\mathrm{e}-\mathrm{t}}$ represents the theoretical amount of extract, i.e. the amount of extract used in the microencapsulation process. $M_{\text {e-ne }}$ corresponds to the non-encapsulated extract remaining after the encapsulation process (determined by HPLC as previously described). Since the extract corresponds to a complex mixture of several components, the two major compounds derived from the ellagic acid, sanguiin and lambertianin were chosen as the model chemical species to be quantified for $\mathrm{EE}$ evaluation purposes.

\section{Incorporation of free and microencapsulated hydroalcoholic extracts of $R$. ulmifolius in a yogurt}

The chosen food matrix was a natural yogurt without added sugar and $3.5 \%(\mathrm{w} / \mathrm{w})$ of fat. The yoghurt $(140 \mathrm{~g})$ was placed in a glass container and mixed in order to ensure homogeneity to produce all the samples needed for the assays. Then, this mass was divided into six portions for the preparation of the following samples: two samples of pure yogurt with $25 \mathrm{~g}$ each (used as the control sample), two samples of yogurt with $25 \mathrm{~g}$ each for free extract incorporation $(6.25 \mathrm{mg}$ of extract in each one) and two samples of yogurt with $20 \mathrm{~g}$ for microencapsulated extract incorporation (40 $\mathrm{mg}$ of lyophilized microspheres in each one). The samples were prepared taking into consideration the use of the same extract/yogurt ratio $\left(0.25 \mathrm{mg} \mathrm{g}^{-1}\right)$.

The antioxidant activity was evaluated at two different sampling times, namely: at initial time $(t=0)$, i.e. immediately after the addition of free or microencapsulated extracts, and after 3 days $(t=3)$. The collected samples at $t=0$ and $t=3$ were then lyophilized and conditioned for future analysis. The antioxidant activity was evaluated by determining the DPPH radical scavenging activity and reducing power. The procedures used are described in previous sections (DPPH radical-scavenging activity and Reducing power).

\section{Statistical analysis}

All the assays were carried out in triplicate and the results are expressed as mean values and standard deviation (SD). The results were analyzed using one-way analysis of variance (ANOVA) followed by Tukey's HSD test with $\alpha=0.05$, performed by using the SPSS v. 18.0 program.

\section{Results and discussion}

\section{Phenolic profile of the hydroalcoholic extract and decoction}

The HPLC phenolic profile of Rubus ulmifolius, obtained after hydroalcoholic extraction, and recorded at 280 and $370 \mathrm{~nm}$ is shown in Fig. 1; peak characteristics, identities and quantification are presented in Table 1. Twenty-four phenolic compounds were identified in both samples, in which seven were identified as phenolic acid derivatives (di- and caffeolyquinic, $p$-coumaroylquinic, feruloylquinic acids and ellagic acid), eleven as flavonoids (quercetin and kaempferol derivatives and catechin), and six as hydrolyzable tannins (lambertianin, sanguiin and four di-hexahydroxydiphenol (HHDP)galloyl glucose isomers).

Quinic acid derivatives were the main phenolic acids identified, according to their UV ( $\lambda_{\max }$ at $314-330 \mathrm{~nm}$ ) and mass spectra (pseudo-molecular ions $[\mathrm{M}-\mathrm{H}]^{-}$at $m / z 337,353$ and 367 , all of them yielding a product ion at $\mathrm{m} / \mathrm{z} 191$, due to the deprotonated quinic acid). Peak 1, the major phenolic acid derivative found, and peak 2 were identified as 3-O-caffeoylquinic acid and 3-p-coumaroylquinic acid, respectively. Peak 1 yielded deprotonated quinic acid $(\mathrm{m} / \mathrm{z}$ at 191) as the base peak and another majority ion corresponding to the hydroxycinnamic acid residue at $m / z 179$ ([caffeic acid $-\mathrm{H}]^{-}$), and peak 2 presented $m / z 163\left([p \text {-coumaric acid }-\mathrm{H}]^{-}\right)$as the base peak, a fragmentation pattern characteristic of the corresponding 3-acylquinic acids according to Clifford et al. ${ }^{16,17}$ Similarly, peak 3 was tentatively identified as 3-O-feruloylquinic acid taking into account its pseudo-molecular ion and fragmentation 

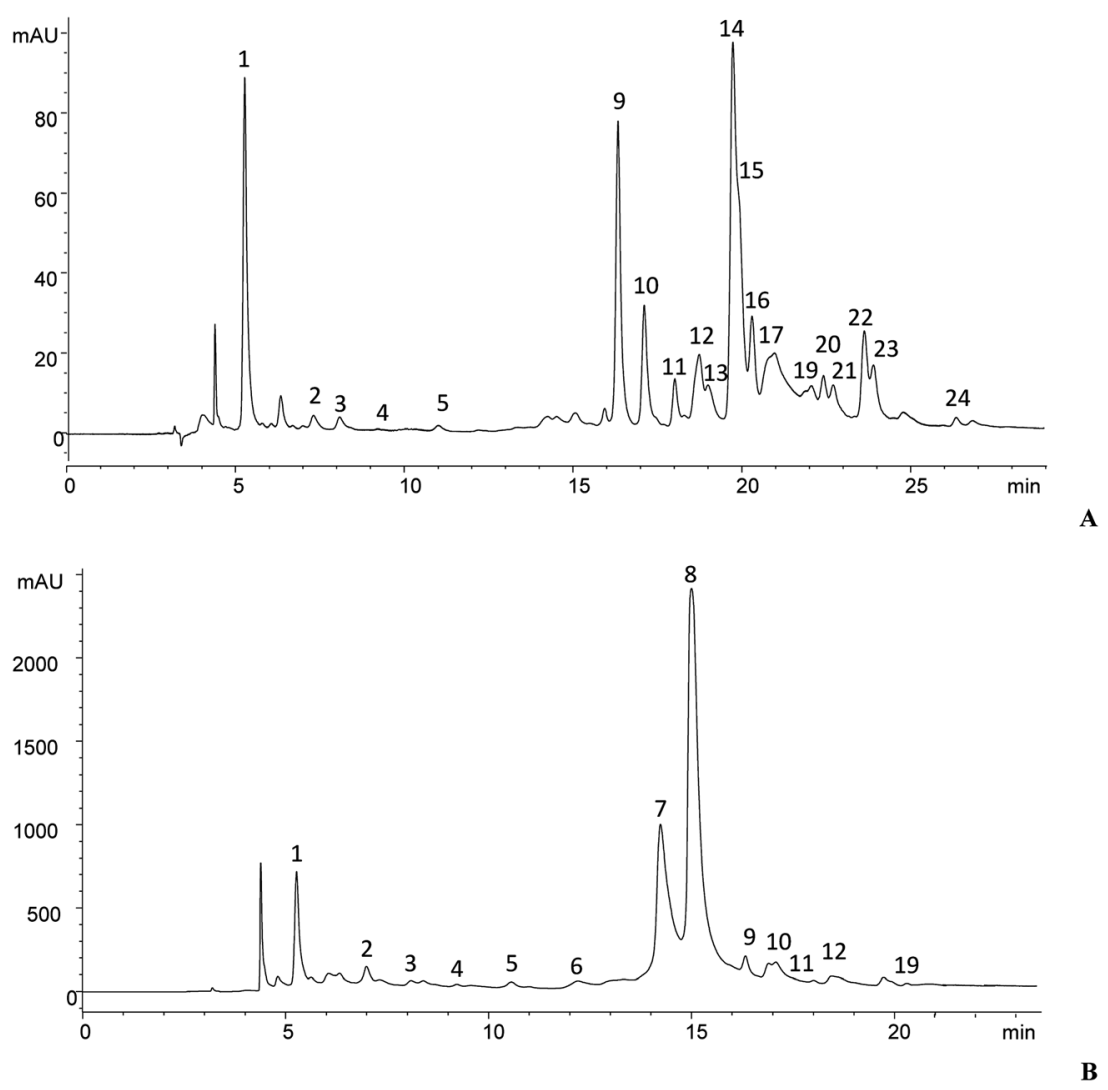

Fig. 1 HPLC phenolic profile of the Rubus ulmifolius hydroalcoholic extract, obtained at $370 \mathrm{~nm}(\mathrm{~A})$ and $280 \mathrm{~nm}$ (B).

pattern, yielding a majority ion at $m / z 193\left([\text { ferulic acid }-\mathrm{H}]^{-}\right.$) as the base peak. Peak $19\left([\mathrm{M}-\mathrm{H}]^{-}\right.$at $\left.m / z 515\right)$ was assigned to 3,5-O-dicaffeoylquinic acid based on its elution order and mass spectra characteristics. The $\mathrm{MS}^{2}$ base peak was at $m / z 353$, produced by the loss of one of the caffeoyl moieties $([\mathrm{M}-\mathrm{H}-$ caffeoyl $]^{-}$), and subsequent fragmentation of this ion yielded the same fragments as 5-caffeoylquinic acid at $m / z 191,179$ and 135 , although in this case with a comparatively more intense signal at $m / z 179$ [caffeic acid $-\mathrm{H}]^{-}(\sim 60 \%$ base peak $) .{ }^{16,17}$ Peak 4 presented a UV spectrum similar to that of $p$-coumaric acid, with $\lambda_{\max }$ around $313 \mathrm{~nm}$; the peak area was very small and did not allow a clear pseudo-molecular ion to be obtained, although signals at $m / z 163\left([\text { coumaric acid }-\mathrm{H}]^{-}\right)$and $m / z 119([$ coumaric acid $\left.-\mathrm{CO}_{2}-\mathrm{H}\right]^{-}$) were observed at its retention time, which allowed them to be assigned as $p$-coumaroyl derivatives.

Peak 18 corresponds to ellagic acid and was positively identified according to its retention, mass and UV-vis characteristics by comparison with the commercial standard; this peak was only found in the decoction preparation.

Regarding flavonoids, mainly flavonol derivatives (Table 1), were found. Catechin (peak 5), quercetin 3-O-rutinoside (peak 13), quercetin 3-O-glucoside (peak 15), kaempferol 3-O-rutinoside (peak 20) and kaempferol 3-O-glucoside (peak 23) were positively identified according to their retention, mass and UVvis characteristics by comparison with commercial standards. Peaks 14 and 16 presented UV spectra with $\lambda_{\max }$ around $350 \mathrm{~nm}$ and an $\mathrm{MS}^{2}$ product ion at $\mathrm{m} / \mathrm{z} 301$ indicating that they correspond to quercetin derivatives. According to their pseudomolecular ions $[\mathrm{M}-\mathrm{H}]^{-}$at $m / z 477$ and 463, they were identified as quercetin 3-O-glucuronide (peak 14), which was confirmed by comparison with a standard isolated in our laboratory, ${ }^{\mathbf{1 8}}$ and a quercetin 3 -O-hexoside (peak 16). Similar reasoning also allowed peaks 21 and 22 to be assigned as kaempferol 3-O-glucuronide and kaempferol 3-O-hexoside, respectively. Peaks 17 and 24 should correspond to kaempferol $O$-pentosyl hexoside and kaempferol $O$-acetylhexoside according to their pseudo-molecular ions $\left([\mathrm{M}-\mathrm{H}]^{-}\right.$at $m / z 579$ and 489 , respectively) and $\mathrm{MS}^{2}$ fragment released at $m / z 285$ (quercetin; [M - H - 132-162 $]^{-}$loss of a pentosyl-hexoside moiety and $[\mathrm{M}-\mathrm{H}-42-162]^{-}$, loss of an acetylhexoside moiety, respectively).

The remaining detected compounds corresponded to hydrolyzable ellagitannins. Peaks 6 and 8 presented the same pseudo-molecular ion $[\mathrm{M}-\mathrm{H}]^{-}$at $m / z$ 1567, which produced $\mathrm{MS}^{2}$ fragment ions at $m / z 1265$ (by the loss of a hexahydroxydiphenoyl group, HHDP), m/z 1103 (loss of HHDP and glucosyl moieties), $m / z 933$ (further loss of a gallate unit), $m / z 631$ (loss of an additional HHDP group) and $m / z 301$ (HHDP released after the final loss of glucosyl-gallate). The signal detected at $\mathrm{m} / \mathrm{z} 783$ would correspond to the pseudo-molecular doubly charged ion $[\mathrm{M}-\mathrm{H}]^{-2}$, as established by zoom scan analysis. These characteristics were coherent with the structure of sanguiin $\mathrm{H}-10$ (Fig. 2b); ${ }^{19,20}$ the observation of two peaks might be due to different configurations in the glucose units, either $\alpha$ or $\beta$, as 
Table 1 Retention time $\left(R_{\mathrm{t}}\right)$, wavelengths of maximum absorption in the visible region $\left(\lambda_{\max }\right)$, mass spectral data, identification and quantification (mg g ${ }^{-1}$ extract or decoction) of phenolic compounds in Rubus ulmifolius ${ }^{a}$

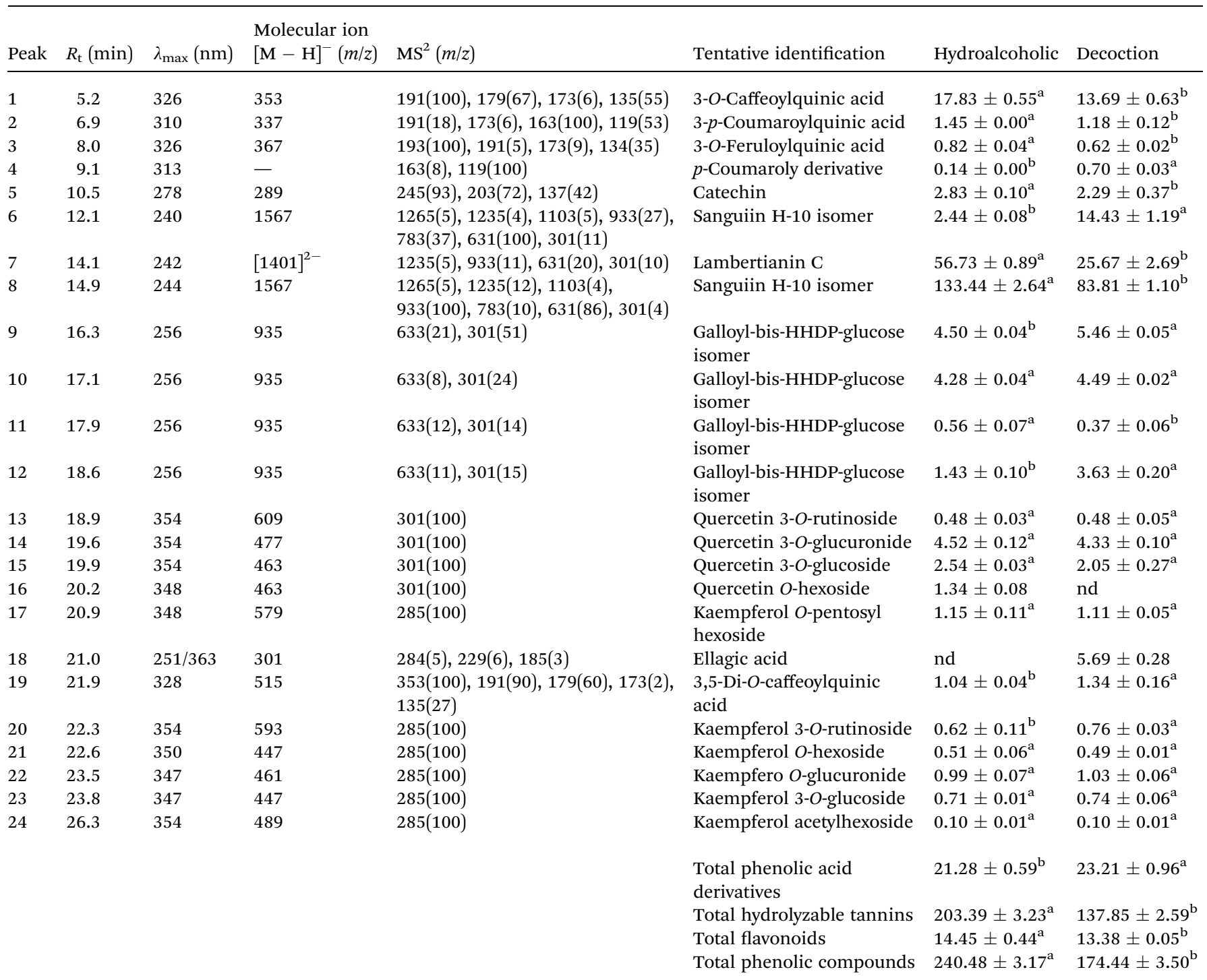

${ }^{a}$ In each row different letters mean significant differences $(p<0.05)$.

previously observed by Kool et al. ${ }^{20}$ Thus, peaks 6 and 8 were identified as sanguiin $\mathrm{H}-10$ isomers. Peak 7 presented a pseudomolecular ion $[\mathrm{M}-\mathrm{H}]^{-2}$ at $m / z 1401$ that was doubly charged as shown by zoom scan analysis and its $\mathrm{MS}^{2}$ fragmentation released singly charged product ions at $m / z$ 1235, 933, 631 and 301 . These characteristics were coherent with the trimeric ellagitannin lambertianin C (Fig. 2c), composed of three galloylbis-HHDP glucose units (molecular mass of $2085.8 \mathrm{Da}$, out of the analyzed $\mathrm{m} / \mathrm{z}$ range), previously described in blackberry fruits ${ }^{21,22}$ and other Rubus species. ${ }^{19,20,23}$ Peaks 9-12 presented a pseudo-molecular ion $[\mathrm{M}-\mathrm{H}]^{-}$at $m / z$ 935, releasing $\mathrm{MS}^{2}$ product ions at $\mathrm{m} / z 633$ and 301, likely due to the loss of HHDP and galloyl-glucose moieties, which is consistent with galloyl-bis-HHDP-glucose ${ }^{21,24}$ and allowed their identification as different galloyl-bis-HHDP glucose isomers (Fig. 2a).
The phenolic profile of both preparations was identical, varying mostly in the concentrations found (Table 1). The hydroalcoholic extract presented higher concentration in total phenolic compounds (240.48 $\mathrm{mg} \mathrm{g}^{-1}$ extract) than the decoction, mainly due to the higher concentration in hydrolyzable tannins (203.39 $\mathrm{mg} \mathrm{g}^{-1}$ extract). The most abundant compounds found in both preparations were ellagitannin derivatives, such as a sanguiin $\mathrm{H}-10$ isomer and lambertianin C. These same compounds have also been reported as relevant phenolic compounds in Rubus fruits, including blackberries, by other authors. ${ }^{19-25}$ Besides ellagitannins, fruits of Rubus species are also known to contain some amounts of flavonoids such as quercetin and kaempferol based flavonol conjugates, with the major components being quercetin 3-O-glucuronide and quercetin 3 -O-glucoside, ${ }^{\mathbf{2 6}}$ as well as ellagic acid, which were also found in the flowers of $R$. ulmifolius studied herein. Ellagic acid 


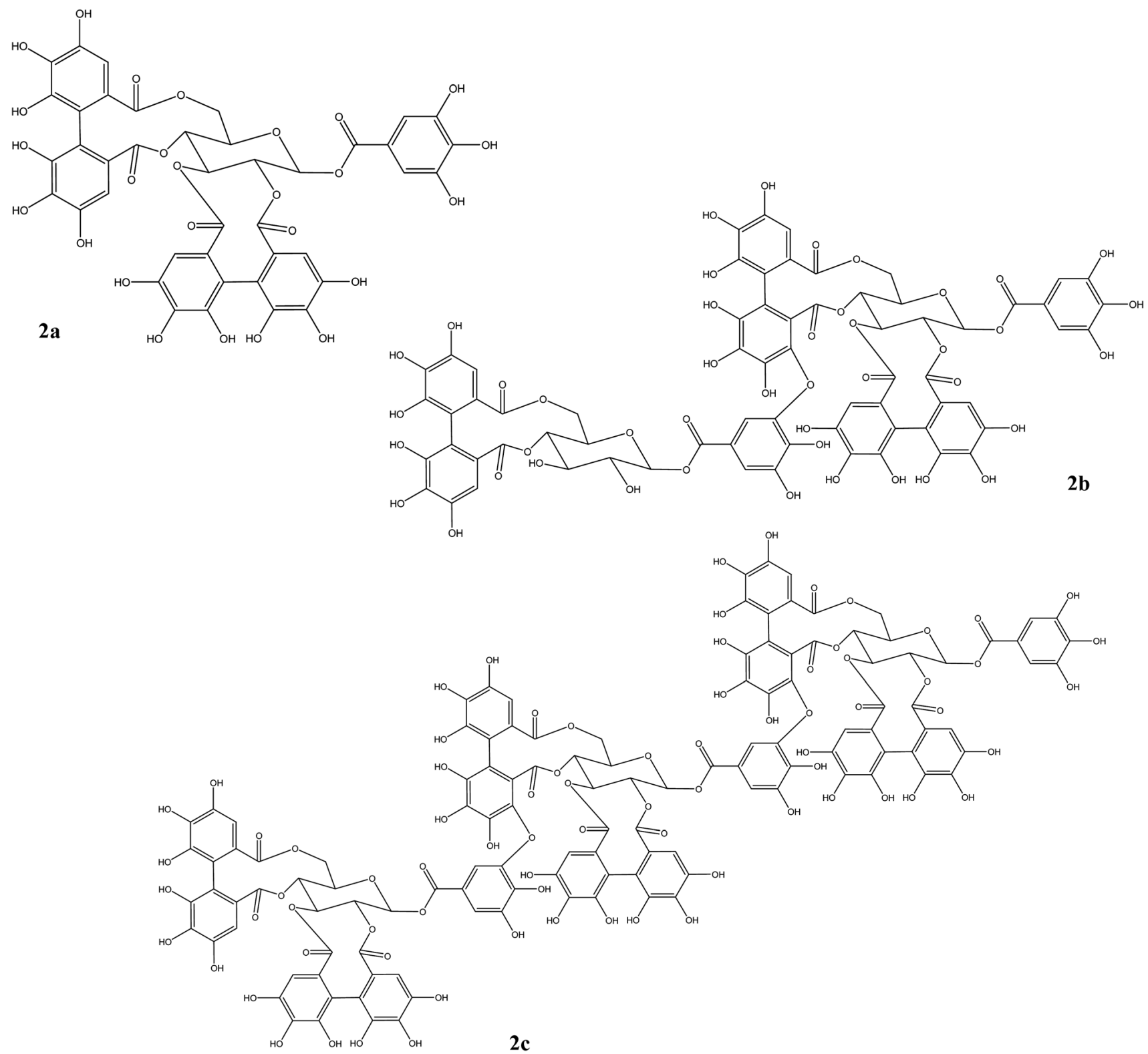

Fig. 2 Structures of ellagitannins found in Rubus ulmifolius, formed by dimers (sanguiin $\mathrm{H}-10$ ) (2b) and trimers (lambertianin C) (2c) of galloylbis-HHDP glucose (2a).

has been reported to have antiviral activity and provide protection against cancers of the colon, lung, and esophagus, and the health benefits of raspberry consumption have been promoted on the basis of claims of a high ellagic acid and ellagitannin content. ${ }^{27}$ To the best of our knowledge, this is the first time that these compounds were identified and quantified in $R$. ulmifolius flower buds.

\section{In vitro antioxidant properties of the hydroalcoholic extract and decoction}

The results obtained in the evaluation of the antioxidant activity of the hydroalcoholic extract and decoction of $R$. ulmifolius are given in Table 2. The hydroalcoholic extract gave higher antioxidant activity (lower $\mathrm{EC}_{50}$ values) in all the in vitro assays
$\left(\mathrm{EC}_{50}\right.$ values between 34.23 and $1.58 \mu \mathrm{g} \mathrm{mL} \mathrm{m}^{-1}$ ) than the decoction preparation $\left(\mathrm{EC}_{50}\right.$ values ranging from 201.72 and 184.21 $\mu \mathrm{g} \mathrm{mL}^{-1}$ ). This is in agreement with the higher phenolic compound concentration $\left(240.48 \mathrm{mg} \mathrm{g}^{-1}\right.$ ) found in the hydroalcoholic extract in comparison to the decoction preparation (177.44 $\mathrm{mg} \mathrm{g}^{-1}$ ).

The methanol extract previously studied by our research group 9 presented slightly higher $\mathrm{EC}_{50}$ values $\left(\leq 40 \mu \mathrm{g} \mathrm{mL} \mathrm{mL}^{-1}\right)$ and, therefore, lower antioxidant activity. There are various studies that report the antioxidant activity of fruits of Rubus species and one specific report ${ }^{28}$ that studied the antioxidant activity of $R$. ulmifolius leaves using ABTS radical decolourisation assay. Nevertheless, to the best of our knowledge, there are no reports available on the decoction preparation of the mentioned plant or its hydroalcoholic extract. 
Table 2 Antioxidant activity of Rubus ulmifolius hydroalcoholic extract and decoction (mean \pm SD) ${ }^{a}$

\begin{tabular}{|c|c|c|}
\hline Antioxidant activity & Hydroalcoholic & Decoction \\
\hline DPPH scavenging activity $\left(\mathrm{EC}_{50}, \mu \mathrm{g} \mathrm{mL} \mathrm{m}^{-1}\right)$ & $34.23 \pm 2.75^{\mathrm{a}}$ & $184.21 \pm 21.40^{\mathrm{b}}$ \\
\hline Reducing power $\left(\mathrm{EC}_{50}, \mu \mathrm{g} \mathrm{\textrm {mL } ^ { - 1 } )}\right.$ & $29.27 \pm 0.80^{\mathrm{a}}$ & $191.23 \pm 0.58^{\mathrm{b}}$ \\
\hline TBARS inhibition $\left(\mathrm{EC}_{50}, \mu \mathrm{g} \mathrm{mL}^{-1}\right)$ & $1.58 \pm 0.07^{\mathrm{a}}$ & $201.72 \pm 3.67^{\mathrm{b}}$ \\
\hline
\end{tabular}

\section{Production of alginate microspheres containing $R$. ulmifolius hydroalcoholic extract}

Alginate-based microspheres containing lyophilized $R$. ulmifolius hydroalcoholic extract were prepared by using an atomization/coagulation technique. The produced microspheres were analyzed by MO immediately after the atomization and 4 hours under the coagulating stage (Fig. 3). Microspheres, in both stages, exhibited a spherical shape and were perfectly individualized without the presence of agglomerates. Their estimated size was between 79 and $380 \mu \mathrm{m}$. In addition, the microspheres presented a lightly homogeneous pink color characteristic of the extract, indicating its incorporation and good distribution inside the microspheres. HPLC analysis of ellagic acid derivatives (sanguiin H-10 and lambertianin C), both in the coagulation and in the first wash solutions, showed that these compounds were present in residual concentrations (below the detection limit) or absent. These data allowed an encapsulation efficiency estimation close to $100 \%$.

\section{Microsphere rehydration after lyophilisation}

The lyophilized microspheres were rehydrated in distilled water for a period of 24 hours in order to test the initial morphology recovery. Fig. 4 shows the OM analysis of the dried and rehydrated microspheres at magnifications of 40,100 and $400 \times$. As it can be seen, the final size of the rehydrated microspheres is close to the one of the initial microspheres (before the lyophilization) showing their good rehydration capacity. Water recovery after 24 hours was $80 \%$ of the originally hydrated microspheres (obtained after production).

\section{Fourier transform infrared spectroscopy (FTIR)}

The FTIR spectra are shown in Fig. 5 (pure alginate, pure extract and microspheres containing the lyophilized extract). As expected, the microsphere spectrum is dominated by the presence of alginate, since an extract/alginate ratio of 100/800 (w/w) was used (see major contributions indicated by the dotted blue line). However, in the microsphere spectrum it is possible to note the contribution from the carbonyl $(\mathrm{C}=\mathrm{O})$ and hydroxyl groups $(\mathrm{OH})$ of the extract (indicated by the dashed red lines). The widening of the $\mathrm{OH}$ and $\mathrm{C}=\mathrm{O}$ bands can be explained by the previous statement and represents evidence for the presence of extract in the microspheres.

\section{Incorporation of free and microencapsulated hydroalcoholic extracts of $R$. ulmifolius in a yogurt}

Table 3 shows the obtained results for the antioxidant activity evaluated according to two parameters: DPPH radical-scavenging activity and reducing power. Both forms (microencapsulated and free extract) exhibited greater activity than the control (lower $\mathrm{EC}_{50}$ values for both DPPH radical scavenging activity and reducing power). The results show that the extract also led to microencapsulated products with better preservation of the antioxidant activity over time (in both tests the $\mathrm{EC}_{50}$ values decreased from time 0 to time 3 days).
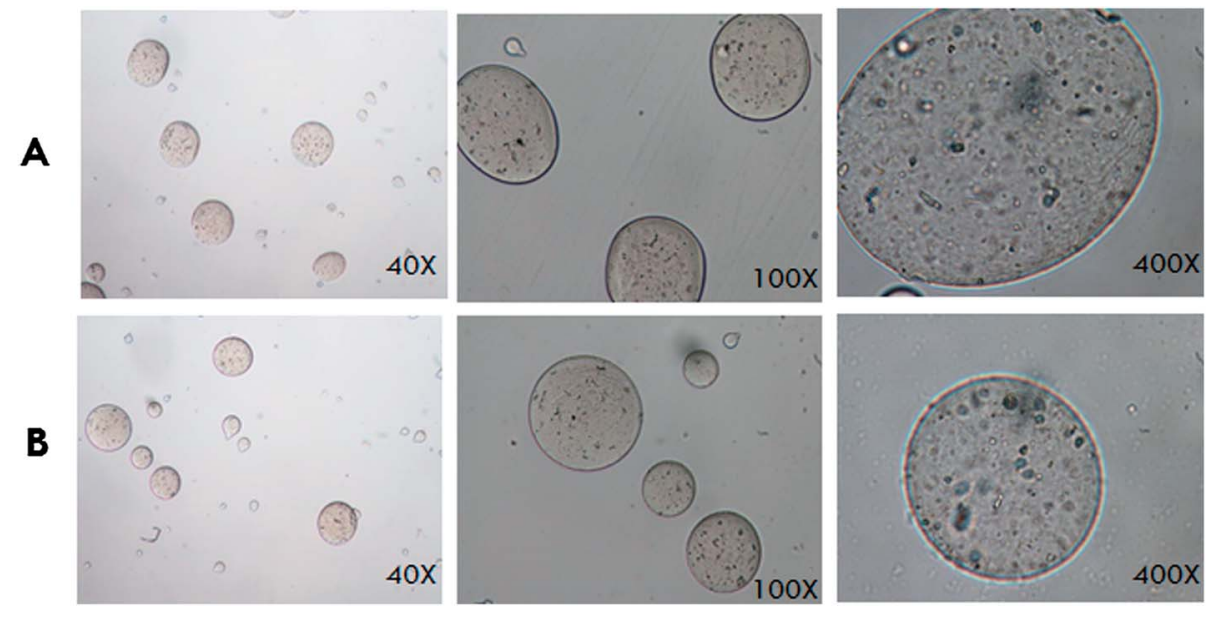

Fig. 3 OM analysis with magnifications of 40,100, $400 \times$ of: the microspheres immediately after atomization (A), and after a 4 hours coagulation period under stirring at $400 \mathrm{rpm}(\mathrm{B})$. 


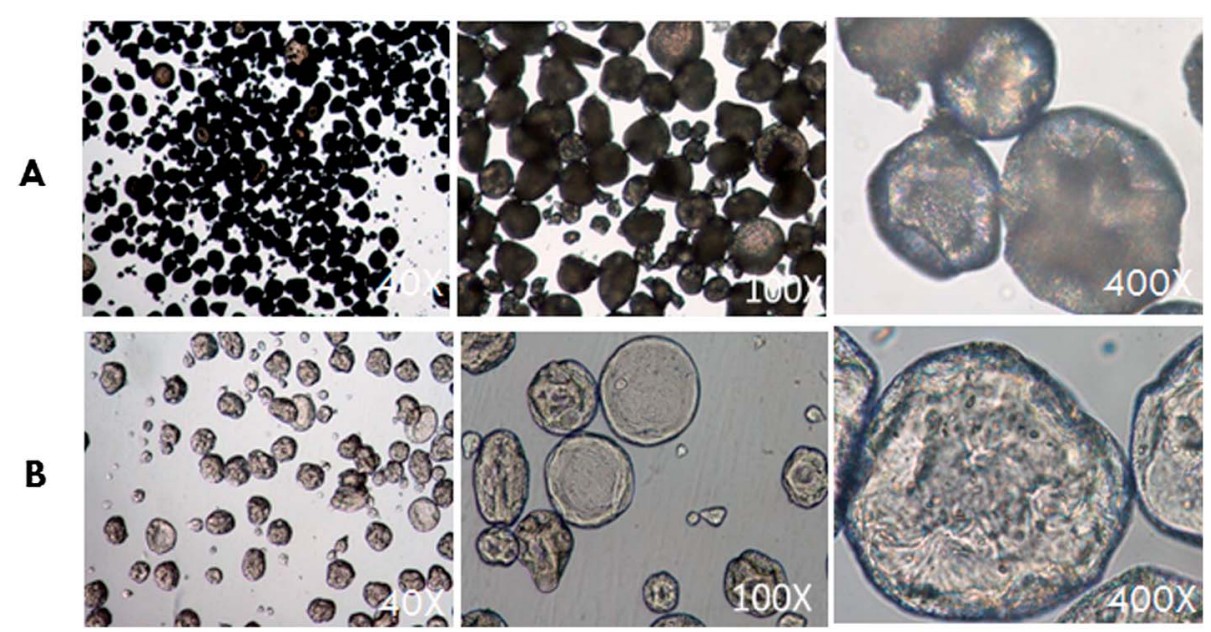

Fig. 4 Microspheres' morphology analysis by OM under magnifications of 40,100 and 400×. (A) Lyophilized microspheres and (B) microspheres after 24 hours of rehydration.

Fig. 6 shows the images of the microspheres incorporated into the yogurt at the initial time $(t=0)$, immediately after the addition of the microencapsulated extract and after 3 days $(t=3)$ at a

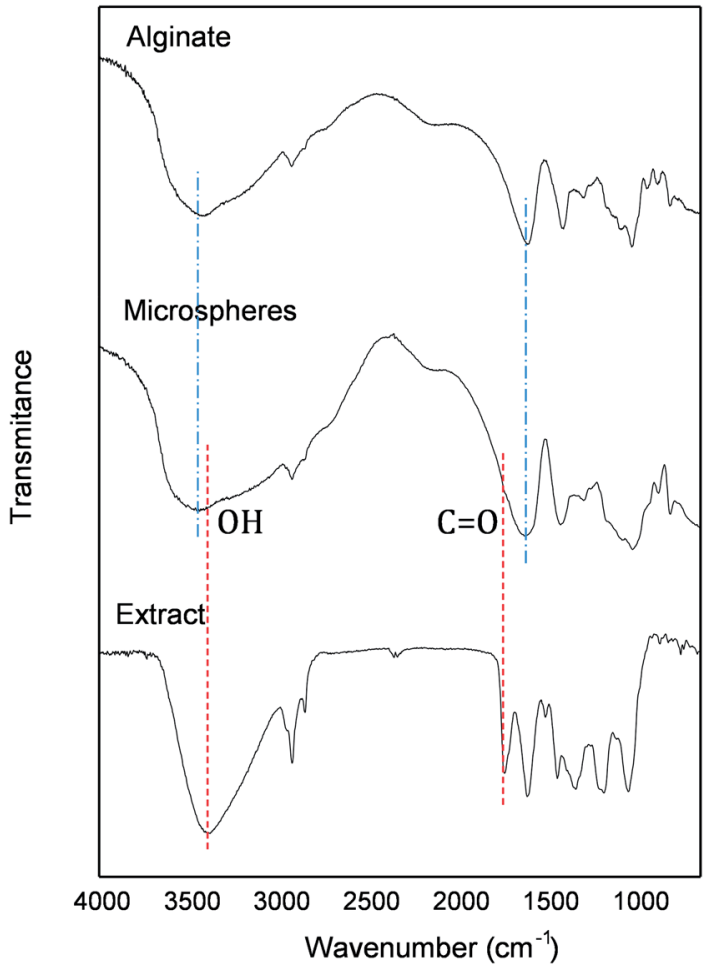

Fig. 5 FTIR spectra of alginate, lyophilized extract and produced microspheres. magnification of 40,100 and 400×. The image analysis for $t=3$ days shows that the microspheres preserve their initial morphology, no microsphere disaggregation was noticed. This fact corroborates also the protective effect of the alginate matrix.

\section{Conclusion}

In summary, the characterization of $R$. ulmifolius flower bud extracts obtained by decoction and hydroalcoholic extraction revealed the presence of twenty-four phenolic compounds, ellagitannin derivatives being the most abundant ones, namely the sanguiin H-10 isomer and lambertianin C. Comparing with the decoction form, the hydroalcoholic extract presented higher antioxidant activity, which can be correlated with its higher phenolic compound content. The atomization/coagulation microencapsulation technique was successfully applied to produce microspheres containing $R$. ulmifolius hydroalcoholic extract which open new avenues for the exploitation of these phenolic extracts in applications such as the food industry. As a preliminary approach the produced microspheres were incorporated into a natural yogurt indicating that, in comparison with its free form, the microencapsulated one is able to better preserve the extract antioxidant activity along time. In summary, the results demonstrated the potential antioxidant activity of the $R$. ulmifolius hydroalcoholic extract and the efficiency of microencapsulation for its preservation.

\section{Competing interests}

The authors declare no competing financial interest.

Table 3 Antioxidant activity of yogurt enriched with the Rubus ulmifolius extract and the microencapsulated extract (mean \pm SD)

\begin{tabular}{|c|c|c|c|c|c|c|}
\hline & \multicolumn{2}{|c|}{ Control yogurt } & \multicolumn{2}{|c|}{ Yogurt with incorporated extract } & \multicolumn{2}{|c|}{ Yogurt with microencapsulated extract } \\
\hline & 0 days & 3 days & 0 days & 3 days & 0 days & 3 days \\
\hline DPPH scavenging activity & $91.19 \pm 1.24$ & $146.17 \pm 5.16$ & $49.34 \pm 0.49$ & $49.88 \pm 2.31$ & $90.71 \pm 3.84$ & $84.15 \pm 1.71$ \\
\hline Reducing power & $2.86 \pm 0.01$ & $13.52 \pm 0.66$ & $16.34 \pm 0.07$ & $2.11 \pm 0.12$ & $15.68 \pm 0.08$ & $1.42 \pm 0.04$ \\
\hline
\end{tabular}


A
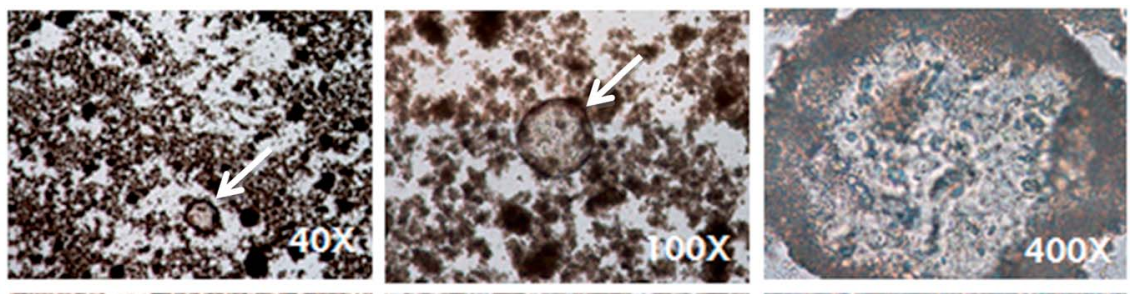

B
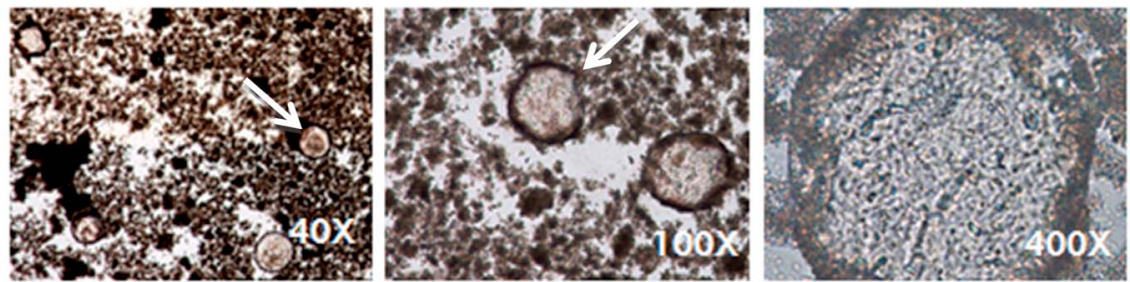

Fig. 6 Microsphere incorporation in a natural yogurt analysis by OM under magnifications of 40,100 and 400×. (A) Microspheres at the initial time $\left(t_{0}\right)$ and $(B)$ microspheres after three days $\left(t_{3}\right)$. The white arrow indicates the incorporated microspheres' structures.

\section{Acknowledgements}

The authors are grateful to Fundação para a Ciência e a Tecnologia (FCT, Portugal) for financial support to CIMO (strategic project PEst-OE/AGR/UI0690/2011) and LSRE (strategic project PEst-C/EQB/LA0020/2011). L. Barros thanks FCT, POPH-QREN and FSE for her researcher contract under "Programa Compromisso com Ciência-2008". The GIP-USAL is financially supported by the Spanish Government through the ConsoliderIngenio 2010 Programme (FUN-C-FOOD, CSD2007-00063).

\section{References}

1 M. Valko, D. Leibfritz, J. Moncol, M. T. D. Cronin, M. Mazur and J. Telser, Int. J. Biochem. Cell Biol., 2007, 39, 44-84.

2 I. C. F. R. Ferreira, L. Barros and R. M. V. Abreu, Curr. Med. Chem., 2009, 16, 1543-1560.

3 M. Carocho and I. C. F. R. Ferreira, Food Chem. Toxicol., 2013, 51, 15-25.

4 Z. Fang and B. Bhandari, Trends Food Sci. Technol., 2010, 21, 510-523.

5 E. Monasterio-Huelin and L. Rubus in S. Castroviejo, (coord. Gen.), S. Flora Iberica, vol VI, Rosaceae, 2001.

6 J. M. Neves, C. Matos, C. Moutinho, G. Queiroz and L. R. Gomes, J. Ethnopharmacol., 2009, 124, 270-283.

7 A. M. Carvalho, Biblioteca de Ciencias, 35, Madrid, Consejo Superior de Investigaciones Científicas, 2010.

8 S. D. Acqua, R. Cervellati, M. C. Loi and G. Innocenti, Food Chem., 2008, 106, 745-749.

9 L. Barros, O. Oliveira, A. M. Carvalho and I. C. F. R. Ferreira, Ind. Crops Prod., 2010, 32, 572-579.

10 V. Nedovic, A. Kalusevic, V. Manojlovic, S. Levic and B. Bugarski, Procedia Food Sci., 2011, 1, 1806-1815.

11 D. Krishnaiah, R. Sarbatly and R. Nithyanandam, Chem. Eng. Res. Des., 2012, 90, 622-632.

12 C. Saénz, S. Tapia, J. Chávez and P. Robert, Food Chem., 2009, 114, 616-622.
13 M. Çam, N. C. Íçyer and F. Erdogan, LWT-Food Sci. Technol., 2013, 55, 117-123.

14 P. N. Ezhilarasi, D. Indrani, B. S. Jena and C. Anandharamakrishnan, J. Food Eng., 2013, 117, 513520.

15 S. Castroviejo (coord), Flora Iberica, vol. VI, Rosaceae, Real Jardín Botánico, Madrid, CSIC, 2001.

16 M. N. Clifford, K. L. Johnston, S. Knight and N. A. Kuhnert, J. Agric. Food Chem., 2003, 51, 2900-2911.

17 M. N. Clifford, S. Knight and N. A. Kuhnert, J. Agric. Food Chem., 2005, 53, 3821-3832.

18 M. Dueñas, H. M. Chornet, J. J. Pérez-Alonso, R. D. PaolaNaranjo, A. M. González-Paramás and C. Santos-Buelga, Eur. Food Res. Technol., 2008, 227, 1069-1076.

19 W. Mullen, T. Yokota, M. E. J. Lean and A. Crozier, Phytochemistry, 2003, 64, 617-624.

20 M. M. Kool, D. J. Comeskey, J. M. Cooney and T. M. McGhie, Food Chem., 2010, 119, 1535-1543.

21 T. J. Hager, L. R. Howard, R. Liyanage, J. O. Lay and R. L. Prior, J. Agric. Food Chem., 2008, 56, 661-669.

22 M. Gasperotti, D. Masuero, U. Vrhovsek, G. Guella and F. Mattivi, J. Agric. Food Chem., 2010, 58, 46024616.

23 W. Mullen, J. McGinn, M. E. Lean, M. R. MacLean, P. Gardner, G. G. Duthie, T. Yokota and A. Crozier, J. Agric. Food Chem., 2002, 50, 5191-5196.

24 K. R. Maatta-Riihinen, A. Kamal-Eldin and A. R. Torronen, J. Agric. Food Chem., 2004, 52, 6178-6187.

25 C. Mertz, V. Cheynier, Z. Gunata and P. Brat, J. Agric. Food Chem., 2007, 55, 8616-8624.

26 P. Zafrilla, F. Ferreres and F. A. Tomás-Barberán, J. Agric. Food Chem., 2001, 49, 3651-3655.

27 R. González-Barrio, G. Borges, W. Mullen and A. J. Crozier, J. Agric. Food Chem., 2010, 58, 3933-3939.

28 S. Martini, C. D'Addario, A. Colacevich, S. Focardi, F. Borghini, A. Santucci, N. Figura and C. Rossi, Int. J. Antimicrob. Agents, 2009, 34, 50-59. 